\title{
Fat within Hydatid Cysts: An Atypical Presentation
}

\author{
H. El Mortaji*, A. Chehboun, M. Benzalim, S. Alj
}

Radiology Department, Ibn Tofail Hospital, Cadi Ayad University, Marrakech, Morocco

DOI: $10.36347 /$ sjmcr.2021.v09i04.022

| Received: 20.02.2021 | Accepted: 13.04.2021 | Published: 24.04.2021

*Corresponding author: $\mathrm{H}$. El Mortaji

\section{Abstract}

The presence of fatty material in a hydatid cyst is a rare and atypical presentation that suggests communication with biliary tree. Few cases have been reported in the literature. Imaging features allow to show the presence of fatty material within the cyst thanks to the density measurement in CT, it also allows to show signs in favor of this communication. We report three cases of hepatic hydatid cysts with fatty component.

Keywords: Hydatid cyst, fat, communication, biliary ducts.

Copyright (C) 2021 The Author(s): This is an open-access article distributed under the terms of the Creative Commons Attribution 4.0 International License (CC BY-NC 4.0) which permits unrestricted use, distribution, and reproduction in any medium for non-commercial use provided the original author and source are credited.

\section{INTRODUCTION}

Hydatid cyst is commonly caused by the larval stage of the tapeworm Echinococcus granulosus. It can occur in any part of the body, but liver is the most commun site of involvement (1). The presence of a fatty component is a very rare complication. It was first reported by Montero and his team in 1996 [2]. They suggested a communication of the hydatid cyst with the biliary tree to explain the fat deposits within the cyst.

\section{Case 1 (Figure 1)}

A 45-year-old female patient presented to our department with intermittent pain in the right hypochondrium associated with jaundice and fever. The abdominal examination revealed a sensitivity of the right hypochondrium. The CT scan identified multiple hydatid cysts of the liver. The one riding segments II and III contained a small fatty area. We also noted a dilation of the neighboring bile ducts which seemed to be in communication with this cyst. The other cysts were multi-partitioned with some wall calcifications.
The communication of the hydatid cyst with the bile ducts was confirmed during the surgical procedure.

\section{Case 2 (Figure 2)}

A 70-year-old male patient presented with pain in the right hypochondrium without associated fever or jaundice. The abdominal computed tomography revealed the presence of a hydatid cyst of the segment VI of the liver containing small drops of fatty density, without dilation of the intrahepatic bile ducts. The patient refused surgery and was put through medical treatment.

\section{Case 3 (Figure 3)}

A 30-year-old male patient admitted to our department as a polytrauma patient. By carrying out his trauma assessment, we accidentally discovered in his abdominal CT a hydatid cyst of the segments II and III of the liver with a fatty component. No dilation of the bile ducts was noted on the radiological assessment. Thought a communication of the cyst with the bile ducts was found on surgery.
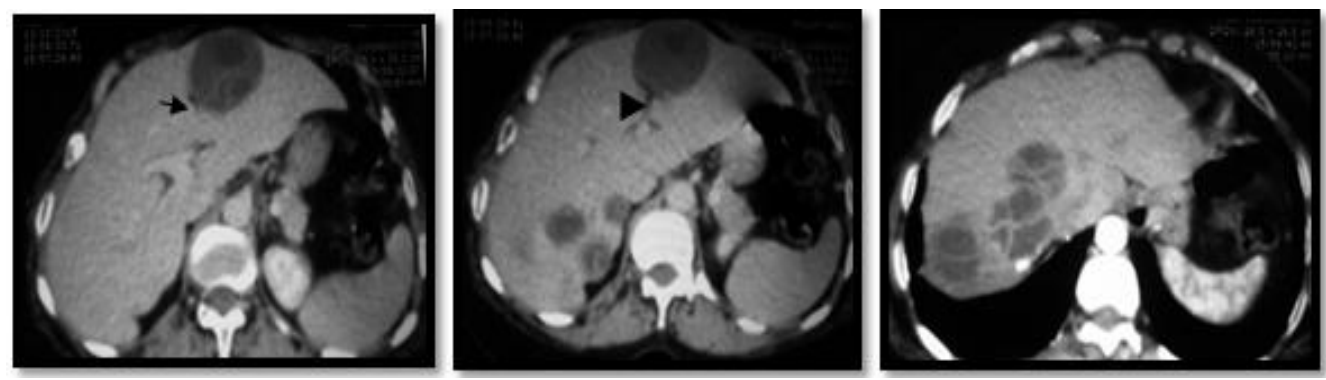

Fig-1: Axial CT images showing hepatic hydatid cysts. The one rinding the segments II and III contains a small fatty area (arrow) with a dilation of the neighboring bile ducts (arrowhead). The other cysts are multi-partitioned and have a partially calcified wall (c) 


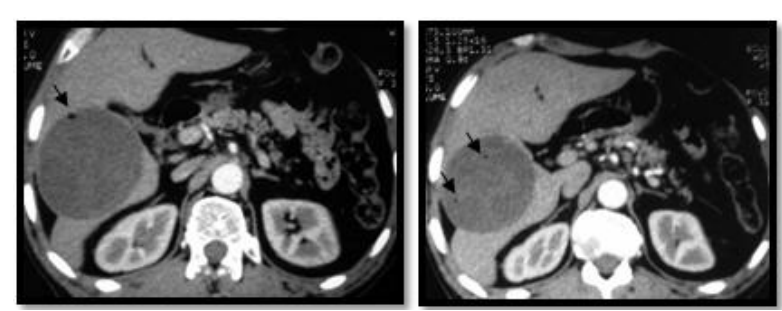

Fig-2: CT axial images showing a large hydatid cyst of segment VI of the liver with a fatty density drops (arrow)
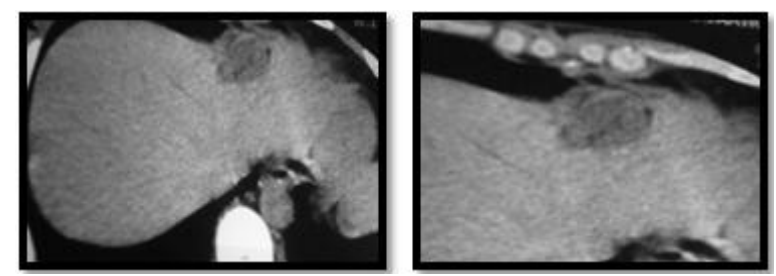

Fig-3: Axial CT images showing a hydatid cyst of the left liver with some fatty drops within. No dilation of bile ducts was shown

\section{DISCUSSION}

Different components usually make up the hydatid cyst: fluid, membranes and calcifications. The association of these different components has given rise to several classifications [3]. The most used one is the classification of Gharbi that includes five evolutionary stages, taking into account the structure of the cystic content, the presence of floating membrane, daughter vesicles or wall calcifications. The presence of a fatty component is not part of the classification.

It is an entity that was described recently. It has been explained by two theories. According to Beric and Blomle [4], the fatty component indicates an aging process of the hydatid cyst. Mendez Montero [2] repported three casent with fatty hepatic hydatid cysts, two of which contained fat-liquid levels, the third case contained granulations of fat density. All of the cases presented with signs of ruptured cysts in the bile ducts. According to this study, the presence of a fatty component inside the hydatid cyst is a very rare sign witnessing cysto-biliary communication allowing the passage of bile which is rich in lipids towards the cystic content [2].

In 2014 AS Tandon and al reported three cases of hepatic hydatid cysts with small fatty granulations diagnosed by computed tomography, only one patient underwent surgery.The cysto-biliary communication was not proven, but the biochemical study showed a hight rate of cholesterol, triglycerides and phospholopids within the hepatic hydatid cyst . According to this study the presence of small fatty granulations within the hydatid cyst can be related to the aging of the hydatid cyst while the presence of a liquid-fat level should make us think and look for a cysto-biliary communication [5].

CT signs indicating a cysto-biliary communication are presented by a dilation of the bile ducts, presence of hydatid material in the bile ducts, or sagging of the cyst. In MRI, the hypersignal on the T1 weighted images within the cyst is a very good sign indicating the presence of a fatty component. However cholangio-MRI is considered to be the gold standard for diagnosing a cysto biliary communication [6].

\section{CONCLUSiON}

The presence on the CT scan of a fatty component within hydatid cysts of the liver is a very rare sign which can be linked to the communication of the cyst with the biliary tree. This is made easy by the discontinuity of the layers of the hydatid cyst leading to its rupture and consequently to the passage of the bile which contains fat towards the hydatid cyst. This entity should be known by radiologist and must lead to look for semiological signs indicating the rupture. Cholangio MRI tend to be very useful and more efficient for this communication in case of doubt.

\section{REFERENCES}

1. Gharbi HA, Hassine W, Brauner M, Dupuch K. Ultrasound examination of the hydatic liver. Radiology. 1981;139:459-63.

2. Montero JVM, Garcia JA, Lafuente JL, Lopez JA, Fernandez RM, Ayala AS. Fat-fluid level in hepatic hydatid cyst: a new sign of rupture into the biliary tree?. AJR American journal of Roentgenol. 1996; 167:91-4. 4.

3. Polat P, Kantarci M, Alper F, Suma S, Koruyucu MB, Okur A. Hydatid disease from head to toe. Radiographics. 2003; 23(2):475-94.

4. Beric V. Fat-fluid level in hepatic hydatid cyst: a new sign of rupture into the biliary tree?. AJR. 1997;168:1381.

5. Tandon AS, Saxena D. Fat within Hepatic Hydatid Cysts: a Report of Three Cases. Hong Kong J Radiol. 2014;17:198-202.

6. Precetti S, Gandon Y, Vilgrain V. Imagerie des lésions kystiques du foie. Journal de radiologie. 2007; 88(7.8). 